\title{
Theorists React to Potential Signal in Dark Matter Detector
}

\author{
A tantalizing signal reported by the XENON1T dark matter experiment has \\ sparked theorists to investigate explanations involving new physics.
}

\author{
By Matteo Rini
}

( n June 16, 2020, the collaboration running XENON1T-one of the world's most sensitive dark matter detectors-reported a signal it couldn't explain (see today's accompanying article, Viewpoint: Dark Matter Detector Delivers Enigmatic Signal). The signal has yet to reach the "5-sigma" bar for discovery, and a mundane explanation could still be the culprit. But theorists have been quick to explore whether exotic particles or interactions might be involved. Physical Review Letters followed a special procedure to get a coherent expert review of the proposals it received. Now, the journal is publishing five papers that represent the breadth of theories being pursued.

All of the reported scenarios explain two aspects of the signal, which was produced in the huge vat of ultrapure xenon that makes up XENON1T's detector. First, the signal looks like it came from particles that collided mostly with the xenon atoms' electrons. And second, each of these interactions dumped a few keV into the atom.

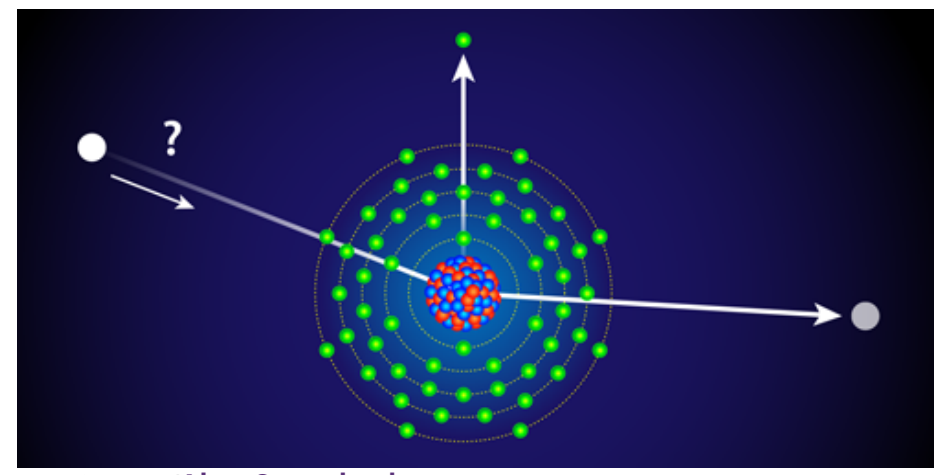

Credit: APS/Alan Stonebraker
Two of the new explanations involve a twist on some of the usual dark matter suspects. Fuminobu Takahashi of Tohoku University, Japan, and colleagues focus on light-mass particles called axions [1]. One problem for axion models is that they assume a coupling between the axions and ordinary photons that is at odds with astrophysical observations. The researchers show that this conflict can be solved-and the XENON1T signal explained-if axions are less likely to interact with photons than most axion models envision. Joseph Bramante and Ningqiang Song of Queen's University, Canada, consider a scenario that involves a low-mass version of the weakly interacting massive particle (WIMP). In their model, a WIMP-like particle scatters into a similar particle with a smaller mass, leaving the mass difference as recoil energy of the xenon electrons [2]. Their scenario is appealing because it's compatible with constraints on dark matter derived from astrophysical observations.

Two of the other ideas share a distinctive feature that XENON1T could spot-a daily modulation of the signal. Nicole Bell of the University of Melbourne, Australia, and colleagues consider "luminous" dark matter particles, which have a "heavy" state and a "light" state separated by about 3 keV [3]. They show that a light-state particle hitting a xenon nucleus could scatter into its heavier counterpart, which then emits a 3-keV photon, whose energy would be dumped onto the electron. Bartosz Fornal of the University of Utah in Salt Lake City and colleagues instead look at "boosted" dark matter [4]. Here, dark matter annihilation processes in the Sun or the Galactic Center would give "cold" dark matter, which normally moves slowly, enough energy to produce the observed keV signal.

Finally, Andreas Bally of the Max Planck Institute for Nuclear Physics, Germany, and co-workers consider a possibility 
involving nonstandard neutrinos [5]. In their model, neutrinos coming from the Sun hit electrons in XENON1T's detector. These collisions could produce the observed signal if some of these neutrinos scattered into nonstandard, "hidden" neutrinos that are invisible to the detectors. Their explanation is associated with beyond-standard-model physics that could be searched for at the Large Hadron Collider and at future accelerators.

Correction (12 October 2020): An earlier version incorrectly said that Bartosz Fornal is at the Chinese Academy of Sciences. $\mathrm{He}$ is at the University of Utah in Salt Lake City.

Matteo Rini is the Deputy Editor of Physics.

\section{REFERENCES}

1. F. Takahashi et al., "XENON1T excess from anomaly-free axionlike dark matter and its implications for stellar cooling anomaly," Phys. Rev. Lett. 125, 161801 (2020).

2. J. Bramante and N. Song, "Electric but not eclectic: Thermal relic dark matter for the XENON1T excess," Phys. Rev. Lett. 125, 161805 (2020).

3. N. F. Bell et al., "Explaining the XENON1T excess with luminous dark matter," Phys. Rev. Lett. 125, 161803 (2020).

4. B. Fornal et al., "Boosted dark matter interpretation of the XENON1T excess," Phys. Rev. Lett. 125, 161804 (2020).

5. A. Bally et al., "Neutrino self-interactions and XENON1T electron recoil excess," Phys. Rev. Lett. 125, 161802 (2020). 